\title{
6 Regionale Netzwerke als Katalysatoren für die digitale Transformation
}

\author{
Leif Grundmann
}

\section{Warum geht das nicht schneller?}

Das Gesundheitswesen in Deutschland hat einen langen Entwicklungs- und Ausgestaltungsprozess hinter sich. Keine andere Wirtschaftsbranche verfügt über ein vergleichbar ausdifferenziertes System, in diesem Fall von Diagnose-, Behandlungs-, Abrechnungs- und Kommunikationsprozessen zwischen vielen kleinen und großen, öffentlichen, freiberuflichen und unternehmerischen Akteuren bis hin zu Patientin und Patient. Das Gesundheitswesen ist daher einerseits die größte Herausforderung für einen erfolgreichen Transformationsprozess, andererseits aber auch ein Anwendungsbereich mit viel ungeborgenem Potenzial für Prozessverbesserungen durch Digitalisierung.

Im Gegensatz zu anderen Branchen bestehen in der Gesundheitsbranche jedoch Rahmenbedingungen, die eine Transformation einbremsen. Getrennte Vergütungsbudgets für den ambulanten und den stationären Sektor, fehlende Finanzierungsanreize für die Anschaffung digitaler Lösungen und ein oftmals ungewisser Return on Investment aufgrund wenig verlässlicher gesetzlicher Rahmenbedingungen machen die Investition in die digitale Zukunft unattraktiv.

\section{Top Down ist keine Lösung}

2002 beschlossen die Spitzenverbände des deutschen Gesundheitswesens eine gemeinsame Vorgehensweise zur Einführung einer elektronischen Gesundheitskarte (eGK), die als Zugangsschlüssel zur bundesweiten Telematikinfrastruktur (TI) den 
Durchbruch zur Digitalisierung des deutschen Gesundheitswesens ermöglichen sollte. 15 Jahre später müssen wir ernüchtert feststellen, dass die TI - (immer noch) auf dem besten Wege der Einführung - zwar eine Autobahn und Verkehrsregeln für die elektronische Kommunikation anbieten wird, die tatsächlichen Anwendungen hingegen noch nicht auf der Straße sind. Neben den unzureichenden Finanzierungsanreizen verhindern Bedenken gegen zu viel Transparenz bei den (dann gläsernen) Leistungserbringern und Patienten, unnötig hohe Hürden beim Datenschutz oder fehlende personelle Ressourcen die breite Einführung von Televisiten, Telemedizin, Decision Support, Zweitmeinungen, digitalem Überleitungsmanagement, elektronischen Fallakten und patientengeführten Gesundheitsakten. Mit den Vorgaben aus dem E-Health-Gesetz der Bundesregierung kommt nun vielleicht neuer Druck auf den Kessel. Dennoch bleibt die Erkenntnis, dass sich die Digitalisierung des Gesundheitswesens nicht Top Down organisieren lässt, sondern in konkreten Teilprojekten, anhand klarer Bedarfe und mit greifbaren Verbesserungen für die umsetzenden Akteure hart erarbeitet werden muss. Nutzerorientierung heißt das Schlüsselwort und bezieht sich in diesem Fall weniger auf die Patienten, als vielmehr auf die professionellen Nutzer im Versorgungsprozess. Werden sie in die Ausgestaltung der neuen Prozesse und Produkte einbezogen, erhöht das die spätere Akzeptanz und auch die Vermarktungschancen.

\section{Transformation regional gestalten}

Für manche Entwicklungen genügt die Zusammenarbeit weniger kompetenter Partner aus Anwendung, Wissenschaft und Industrie. Sobald jedoch Prozesse zwischen mehreren Leistungserbringern auf stationärer oder ambulanter Ebene oder sektorübergreifend digital abgebildet werden sollen, sind maßgeschneiderte Akteurskonstellationen mit mehreren, ideal passenden Partnern gefragt, die entlang einer bestehenden Wertschöpfungs- oder hier Versorgungskette arbeiten.

Folgt man der Argumentation, dass nennenswerte Beiträge zur digitalen Transformation in der Regel

1. vom Bedarf ausgehen (Nutzerorientierung),

2. in Projekten mit vielen/allen Beteiligten erarbeitet werden (Netzwerke) und

3. in bestehenden Akteurskonstellationen bzw. Wertschöpfungsbeziehungen sektorübergreifend eingeführt werden (Referenzregionen),

dann erklärt sich die Bedeutung regionaler Netzwerke für Innovationen. Regional öffnet den Raum insoweit, dass Projekte nicht an Stadt- oder Kreisgrenzen gebunden sein müssen, sondern die funktional relevanten Akteure und Beziehungen unabhängig von politischen, geografischen oder administrativen Grenzen berücksichtigt werden können. Regional begrenzt den Raum der Vorhaben andererseits auf eine Entfernung, die regelmäßige Treffen der Akteure für Erfahrungsaustausch, Projektarbeit oder Workshops ermöglicht. Virtuelle Meetings können den realen Austausch in diesem Falle nicht ersetzen. Persönliche Teilnahme dient der Vertrauensbildung und damit auch nachhaltiger Zusammenarbeit.

An der Ruhr arbeiten bereits seit 2002 zahlreiche Einrichtungen unter der Dachmarke MedEcon Ruhr - Netzwerk der Gesundheitswirtschaft an der Ruhr an städte- und sektor- 
übergreifenden Projekten der Gesundheitswirtschaft zusammen. Seit 2007 steht mit dem gleichnamigen Verein und der $\mathrm{GmbH}$ (als Geschäftsstelle) eine feste Struktur für die Initiierung und Durchführung regionaler Kooperationsprojekte zur Verfügung. Die räumliche Fokussierung auf den Wirkungskreis Metropole Ruhr bringt kaum Nachteile im Sinne einer Beschränkung mit sich. Der polyzentrische Ballungsraum mit mehr als 5 Millionen Einwohnern verfügt in vielerlei Hinsicht über kompetente Partner aus der gesundheitsbezogenen Wissenschaft, Versorgung, Wirtschaft und Bildung. Die Fokussierung auf die Region ermöglicht der Netzwerkeinrichtung (hier aufgrund der räumlichen Verdichtung) jedoch immer noch eine reale Präsenz und die Kenntnis der Akteursstrukturen vor Ort.

Rund 150 Einrichtungen aus Klinikwirtschaft und Gesundheitsversorgung, Lebenswissenschaften und Versorgungsforschung, kommunalen Einrichtungen und zuliefernden Branchen sind über ihre Mitgliedschaft im MedEcon Ruhr e.V. organisiert. Mit der MedEcon Ruhr GmbH hat sich der Verein eine gut aufgestellte Geschäftsstelle mit derzeit 14 Mitarbeiterinnen und Mitarbeitern geschaffen. Sie betreut regionale Netzwerke und Verbünde, entwickelt und koordiniert Projekte, unterstützt und organisiert Veranstaltungen und sorgt mit ihren Medien für Transparenz und Information (www.medecon.ruhr).

\section{Regionale Netzwerke als Basis für konkrete Projekte}

Für die Umsetzung von konkreten Digitalisierungsprojekten werden in aller Regel bestehende Prozesse zugrunde gelegt, analysiert, zerlegt, bewertet und dann Schritt für Schritt digitalisiert und in einer Prozesskette neu zusammengestellt. Zwei Fallbeispiele sollen die Rolle regionaler Netzwerke als Katalysatoren für derartige Projekte veranschaulichen.

\section{Fallbeispiel 1: Westdeutscher Teleradiologieverbund (TRV)}

Radiologen der Region, die bereits in Befundungs- und Behandlungsbeziehungen zusammenarbeiten, treffen sich Ende 2009 um über die Möglichkeit des digitalen Bildaustausches zu sprechen. Selbst tief im Emailzeitalter ist die Übermittlung dieser großvolumigen und behandlungsbezogenen Dateien mit sensiblen Inhalten weder technisch, noch organisatorisch oder sicher gelöst. Auf der neutralen Basis des regionalen Netzwerks (MedEcon Ruhr) werden die Anforderungen und Prozesse formuliert und mit einem geeigneten ansässigen Industriepartner technisch und organisatorisch gelöst. Nach einer einführenden Projektphase startet 2012 der Teleradiologieverbund Ruhr seinen Regelbetrieb und eine Managementgesellschaft wird gegründet. In den kommenden Jahren dienen regelmäßige Anwendertreffen zur nutzerorientierten Weiterentwicklung der angebotenen Dienstleistungen. Anfang 2017 verbindet der mittlerweile größte deutsche Teleradiologieverbund mehr als 280 Krankenhäuser und radiologische Praxen. Weit über die Geburtsregion Ruhr hinaus können Röntgenbilder oder ganze CT- oder MR-Studien heute im Notfall, bei Verlegungen oder Konsilanfragen in wenigen Minuten versendet und empfangen werden - auch bei unterschiedlichsten Quell- und Empfängersystemen. Der konkrete 
Nutzen für die Patienten liegt auf der Hand. Aber auch die an der Entwicklung beteiligten Anwender haben - früher als andere - eine hilfreiche technische Unterstützung für ihre tägliche Arbeit erhalten und stehen mit positiven Erfahrungen für die weitere Entwicklung oder neue Digitalisierungsprojekte zur Verfügung (www. medecon-telemedizin.de).

\section{Fallbeispiel 2: FALKO.NRW}

Ausgehend von der positiven Erfahrung beim Versand radiologischer Bilddaten formulieren die medizinischen Vertreter bei den Anwendertreffen des TRV 2015 einen weitergehenden Bedarf: Zusätzlich zum Bild sollen komplette Falldaten bei Verlegungen oder Konsilanfragen kommuniziert werden. Selbst moderne Krankenhäuser sind heute noch nicht in der Lage alle relevanten Behandlungsdaten zu einem Fall digital für den Versand bereit zu stellen. Gelingt die Falldatenkonsolidierung und der Versand, kann die abweichende Systemkonstellation beim Empfänger die Dokumente nicht ins eigene System übernehmen oder komfortabel für die Weiterbehandlung anbieten. Zur Lösung dieser Problematik organisierte ein regionales Konsortium aus 16 Partnereinrichtungen einen gemeinsamen Lösungsansatz. Das Projekt FALKO. NRW - medizinische Falldatenkommunikation in interoperablen Netzwerken wurde im März 2016 mit Fördergeldern des Landes NRW und der EU gestartet. Ziel ist der Aufbau einer herstellerunabhängigen elektronischen Kommunikationsstruktur für medizinische Falldaten, die nach regionaler Entwicklung und Testung NRW- und bundesweit ausrollbar sein wird. Bereits nach dem ersten Projektjahr sind konkrete Lösungen absehbar und die Projektbeteiligten denken bereits über die gemeinsame Lösung der nächsten Fragestellungen nach (www.falko.nrw).

\section{Regionale Netzwerke sind Prozessbeschleuniger bei der Digitalen Transforma-} tion im Gesundheitswesen

1. Die digitale Transformation im Gesundheitswesen findet nicht Top Down statt.

2. Regional organisierte Umsetzungsprojekte haben das Potenzial, konkret am Bedarf und mit Akteuren bestehender Behandlungsbeziehungen nutzbringende Transformationsschritte zu erarbeiten.

3. Die Relevanz regionaler Projekte bietet Politik und Industrie bei Erfolg die Option des bundesweiten Roll-outs.

4. Regionale Akteurskonstellationen bieten bei nachhaltiger Zusammenarbeit die Basis für immer neue Projektkonstellationen.

\section{Digitale Transformation im Gesundheitswesen - Aber sicher!}

Für die Zukunft werden sich bei fortschreitender Digitalisierung des Cesundheitswesens über die elektronische Abbildung bestehender Behandlungspfade hinaus auch vollkommen neue Aufgabenstellungen ergeben. Die Vernetzung aller Systeme im Krankenhaus, vom Krankenhausinformationssystem (KIS) und die angebundene Medizintechnik über die Haustechnik bis hin zur Verwaltung wird in naher Zukunft realisiert werden, hin zum Krankenhaus 4.o. Welche Herausforderung aber wird die 
sektorübergreifende Kommunikation von Daten der Kassen, des ambulanten Sektor und letztlich auch des Patienten bringen? Bereits heute sind Krankenhäuser attraktive Ziele von Cyberangriffen. Die Sabotage lebenswichtiger Versorgungsinfrastrukturen oder der Diebstahl personenbezogener Behandlungsdaten dienen als Ceschäftsmodell für die Erpressung 4.o. Auch für diesen Themenkreis eignen sich regionale Netzwerke zur Entwicklung und Einführung neuer Lösungen. In der Metropole Ruhr bestehen deutschlandweit führende Kompetenzen im Bereich der IT-Sicherheit in Ausbildung, Forschung und bei Leistungsanbietern. Was liegt also näher als die Vernetzung der Akteure aus IT-Security und Gesundheitsbranche zur Entwicklung nutzerorientierter Sicherheitslösungen für ein digitales Gesundheitswesen? Die ersten Verbundprojekte sind bereits in Arbeit. 